\title{
A HOMOTOPY THEOREM FOR MATROIDS, II
}

\author{
BY \\ W. T. TUTTE
}

1. Introduction. In this paper $R$ denotes a ring, which is either the ring of integers or the ring of residues modulo a prime.

If $M$ is any finite set we define a chain on $M$ over $R$ as a mapping $f$ of $M$ into $R$. If $a \in M$ then $f(a)$ is the coefficient of $a$ in $f$. The set of all $a \in M$ such that $f(a) \neq 0$ is the domain $|f|$ of $f$. If $f(a)=0$ for all $a$ then $f$ is the zero chain on $M$ over $R$. We denote a zero chain by the symbol 0 .

The sum $f+g$ of two chains $f$ and $g$ on $M$ over $R$ is a chain on $M$ over $R$ defined by the following rule:

$$
(f+g)(a)=f(a)+g(a), \quad a \in M .
$$

With this definition of addition the chains on $M$ over $R$ are the elements of an additive Abelian group $A(M, R)$. Its zero element is the zero chain on $M$ over $R$ and the negative of a chain $f$ is obtained by multiplying each coefficient in $f$ by -1 . We define a chain-group on $M$ over $R$ as any subgroup of $A(M, R)$.

Let $N$ be any chain-group on $M$ over $R$. Let $N^{*}$ be the class of all chains $g$ on $M$ over $R$ such that

$$
\sum_{a \in M} f(a) g(a)=0
$$

for all $f \in N$. Then $N^{*}$ is a chain-group on $M$ over $R$. We call it the dual chain-group of $N$.

A chain $f$ of $N$ is elementary if it is nonzero and there is no nonzero $g \in N$ such that $|g|$ is a proper subset of $|f|$. If $R$ is the ring of integers we define a primitive chain of $N$ as an elementary chain $f$ of $N$ in which the coefficients $f(a)$ are restricted to the values 0,1 and -1 .

We call $N$ binary if $R$ is the ring of residues mod 2. We call it regular if $R$ is the ring of integers and to each elementary chain there corresponds a primitive chain with the same domain. Regular chain-groups are studied in [2]. In that paper it is shown that the groups of integral cycles and coboundaries on an oriented graph are regular chain-groups.

We write $\boldsymbol{M}(N)$ for the class of domains of elementary chains of $N$. We shall show that $\boldsymbol{M}(N)$ is a matroid on $M$. We call it the matroid of $N$. We call a matroid binary or regular if it is the matroid of a binary or regular chain-group respectively. This definition of a binary matroid is clearly equivalent to that given in the Introduction to Paper I [3].

Received by the editors October 26, 1956. 
In this paper we investigate the following problem. What are the necessary and sufficient conditions, in terms of geometrical structure, that a given matroid shall be (a) binary or (b) regular? The problem of binary matroids is found to present little difficulty; the condition is simply that there shall be just three points on each connected line. For regular matroids we need the homotopy theorem proved in Paper I. The conditions are that the given matroid shall be binary and that two particular figures shall not occur in its geometrical structure. One of these excluded figures is the Fano configuration of seven lines and seven points in a plane. The second is the matroid dual of the Fano configuration. This means that the two figures represent the matroids of two binary chain-groups each of which is the dual of the other.

The two theorems just quoted are members of an interesting sequence. The third member, with which I hope to deal in another paper, gives the conditions for a given regular chain-group to be the cycle-group or coboundary-group of a graph. The fourth, a famous theorem of Kuratowski [1] gives the conditions for a given graph to be a planar graph. In each case the condition imposed is equivalent to the exclusion of certain geometrical figures from the corresponding matroid.

2. Chain-groups and matroids. Let $N$ be any chain-group on a set $M$ over $R$. If $r \in R$ and $f \in N$ we define a chain $r f$ on $M$ over $R$ by the following rule:

$$
(r f)(a)=r \times f(a), \quad a \in M .
$$

By the definition of $R$ the chain $r f$ can always be represented as a sum of chains, each equal to $f$ or $-f$. Hence $r f$ is always a chain of $N$.

(2.2) If $f \in N$ and $a \in|f|$ then there exists an elementary chain $g$ of $N$ such that $a \in|g| \subseteq|f|$.

Proof. If possible choose $f$ and $a$ so that the theorem fails and $|f|$ has the least number of elements consistent with this. Since $f$ is nonzero there is an elementary chain $g$ of $N$ such that $|g| \subseteq|f|$. We must have $a \notin|g|$. Choose $b \in|g|$ and write $k=g(b) f-f(b) g$. Then $a \in|k| \subset|f|$. Hence, by the definition of $f$, there exists an elementary chain $g^{\prime}$ of $N$ such that $a \in\left|g^{\prime}\right| \subseteq|k|$. But then $a \in\left|g^{\prime}\right| \subset|f|$, which is impossible.

(2.3) The class $M(N)$ of domains of elementary chains of $N$ is a matroid on $M$.

Proof. We must show that $\boldsymbol{M}(N)$ satisfies Axioms I and II of the Introduction to Paper I. It satisfies Axiom I by the definition of an elementary chain. To verify Axiom II suppose $(X, Y) \in M(N), a \in X \cap Y$ and $b \in X-(X \cap Y)$. There are chains $f$ and $g$ of $N$ such that $|f|=X$ and $|g|=Y$. We have $b$ $\in|g(a) f-f(a) g| \subseteq(X \cup Y)-\{a\}$. Hence, by (2.2), there exists $Z \in M(N)$ such that $b \in Z \subseteq(X \cup Y)-\{a\}$. Thus Axiom II holds for $\boldsymbol{M}(N)$.

As stated in the Introduction we call $\boldsymbol{M}(N)$ the matroid of $N$. It follows from (2.2) that the domain of any chain of $N$ is a flat of $\boldsymbol{M}(N)$. 
Chains $f_{1}, \cdots, f_{k}$ of $N$ are linearly dependent if they satisfy a relation $r_{1} f_{1}+\cdots+r_{k} f_{k}=0$ in which the coefficients $r_{i}$ are elements of $R$ not all zero. If no such relation holds the chains are linearly independent. The $\operatorname{rank} r(N)$ of $N$ is the maximum number of linearly independent chains of $N$.

(2.4) Let $S$ be a flat of $\boldsymbol{M}(N)$ of dimension $d S$. Then the maximum number of linearly independent chains of $N$ with domains in $S$ is $d S+1$.

Proof. If possible choose $S$ so that the theorem fails and $d S$ has the least value consistent with this. Then $d S \geqq 0$ for otherwise $S$ is null and the theorem is trivially true.

Choose $a \in S$. Since $S$ is a flat of $\boldsymbol{M}(N)$ there exists a chain $f$ of $N$ such that $a \in|f| \subseteq S$. But $d\langle S-\{a\}\rangle=d S-1$, by $\mathrm{I}(2.3)$. Hence, by the choice of $S$, there exist $d S$ linearly independent chains of $N$ with domains in $\langle S-\{a\}\rangle$. Adjoining $f$ to these we obtain $d S+1$ linearly independent chains of $N$ with domains in $S$.

We deduce that there exist $k=d S+2$ linearly independent chains $f_{1}, \cdots, f_{k}$ of $N$ with domains in $S$. At most $k-2$ of them have domains in $\langle S-\{a\}\rangle$. We can adjust the notation so that $a \in\left|f_{i}\right|$ if $i \leqq s$ and $a \notin\left|f_{i}\right|$ if $i>s$, where $s$ is some integer satisfying $2 \leqq s \leqq k$. For each $i \leqq s$ we write $g_{i}=f_{1}(a) f_{i}-f_{i}(a) f_{1}$. Then $\left|g_{i}\right| \subseteq\langle S-\{a\}\rangle$. By the choice of $S$ we can find elements $r_{2}, \cdots, r_{k}$ of $R$, not all zero, such that

$$
\sum_{i=2}^{s} r_{i} g_{i}+\sum_{i=s+1}^{k} r_{i} f_{i}=0
$$

Writing the left hand side of this equation in terms of the $k$ chains $f_{i}$ we find that these chains are not linearly independent. This contradiction establishes the theorem.

(2.5) Let $M$ be a matroid on a set $M$. With each $X \in M$ let there be associated a chain $f(X)$ on $M$ over $R$ so that the following conditions are satisfied:

(i) $|f(X)|=X$,

(ii) If $X, Y$ and $Z$ are distinct points on the same connected line of $M$ there exist nonzero elements $r, s$ and $t$ of $R$ such that $r f(X)+s f(Y)+t f(Z)=0$.

Let $N$ be the chain-group on $M$ generated by the chains $f(X)$.

Then $\boldsymbol{M}=\boldsymbol{M}(N)$.

Proof. Let $T$ be any member of $\boldsymbol{M}(N)$. There exists $f \in N$ such that $|f|$ $=T$. By the definition of $N$ we can find a flat $S$ of $M$ with the following properties:

(i) There is a nonzero $r \in R$ such that $r f$ is a sum of chains $\pm f(X)$ with $X \subseteq S$,

(ii) $d S$ has the least value consistent with (i).

If possible choose $a \in S-T$. Write $S^{\prime}=\langle S-\{a\}\rangle$. By I(2.2) any line of $\boldsymbol{M}$ which is not a subset of $S^{\prime}$ has at most one point in common with $S^{\prime}$. Hence the class $C$ of all points of $M$ which are subsets of $S^{\prime}$ is a convex subclass of $M$. 
If $g$ and $h$ are chains of $N$ we write $g \equiv h \bmod S^{\prime}$ to denote that $g-h$ is a sum of chains $\pm f(X)$ such that $X \subseteq S^{\prime}$. In particular we may write

$$
r f \equiv \sum_{i} \pm f\left(Y_{i}\right) \bmod S^{\prime},
$$

where the $Y_{i}$ are points of $\boldsymbol{M}$ satisfying $a \in Y_{i} \subseteq S$.

Choose a point $Y$ of $\boldsymbol{M}$ such that $a \in Y \subseteq S$. Then for each $Y_{i}$ the flat $Y \cup Y_{i}$ is connected. Hence, by $\mathrm{I}(5.1)$, there is a path $P_{i}$ from $Y_{i}$ to $Y$ on $S$ whose terms are all points of $\boldsymbol{M}-\boldsymbol{C}$. If $U$ and $V$ are consecutive terms of $P_{\boldsymbol{i}}$ then the line $U \cup V$ meets $S^{\prime}$ in a point $W$ of $C$, by $\mathrm{I}(2.5)$, and by hypothesis we have

$$
x_{i} f(U) \equiv y_{i} f(V) \bmod S^{\prime},
$$

where $x_{i}$ and $y_{i}$ are nonzero members of $R$. If $Y_{i} \neq 1$ we obtain (2.5b) for each pair of consecutive terms of $P_{i}$ and combine the results. We thus obtain

$$
r_{i} f\left(Y_{i}\right) \equiv s_{i} f(Y) \bmod S^{\prime}
$$

where $r_{i}$ and $s_{i}$ are nonzero elements of $R$. Multiplying (2.5a) by the product of the elements $r_{i}$ we obtain

$$
r^{\prime} f \equiv s^{\prime} f(Y) \bmod S^{\prime}
$$

where $r^{\prime}$ and $s^{\prime}$ are elements of $R, r^{\prime}$ being nonzero.

Since $a \notin|f|$ we must have $s^{\prime}=0$. Then (2.5d) contradicts the definition of $S$. We deduce that $S=T$. Hence there exists $T^{\prime} \in M$, on $S$, such that $T^{\prime} \subseteq T$.

Conversely let $T^{\prime}$ be any member of $\boldsymbol{M}$. Then $f\left(T^{\prime}\right) \in N$. Hence there exists $T \in M(N)$ such that $T \subseteq T^{\prime}$.

Applying Axiom I to these results we find that the classes $\boldsymbol{M}$ and $\boldsymbol{M}(N)$ are identical.

(2.6) Let $\boldsymbol{M}$ be a matroid, and $L$ a line of $\boldsymbol{M}$ on which there are just three points, $X, Y$ and $Z$. Then each cell of $L$ belongs to just two of the points $X, Y$ and $Z$.

Proof. Let $a$ be any cell of $L$. By I(3.1) we have $L=X \cup Y=Y \cup Z$ $=Z \cup X$. Hence $a$ belongs to at least two of the points on $L$. But if $a$ belongs to all three then, by $\mathrm{I}(2.3)$, the flat $\langle L-\{a\}\rangle$ is a point on $L$ distinct from $X, Y$ and $Z$.

(2.7) $A$ matroid $\boldsymbol{M}$ is binary if and only if there are just three points on each connected line of $\boldsymbol{M}$.

Proof. $M$ is a matroid on a set $M$. If $S \subseteq M$ we define $f(S)$ as the chain on $M$ over the ring of residues $\bmod 2$ such that $f(a)=1$ if $a \in S$ and $f(a)=0$ if $a \in M-S$.

Suppose $\boldsymbol{M}$ is binary. Let $L$ be any connected line of $\boldsymbol{M}$. There are three distinct points $X, Y$ and $Z$ on $L$, by I(3.2). If $T$ is a fourth point on $L$ we have $f(X)+f(Y)+f(Z)=0$ and $f(X)+f(Y)+f(T)=0$, by (2.4). But then 
$f(Z)=f(T)$ and $Z=T$, contrary to the definition of $T$.

Conversely suppose there are just three points on each connected line of $\boldsymbol{M}$. Then if $X, Y$ and $Z$ are the three points on any connected line $L$ we have $f(X)+f(Y)+f(Z)=0$, by (2.6). Hence $\boldsymbol{M}=\boldsymbol{M}(N)$, by (2.5), where $N$ is the binary chain-group on $\boldsymbol{M}$ generated by those chains $f(S)$ for which $S \in M$. Thus $M$ is a binary matroid.

3. Some operations on matroids. Let $M$ be any matroid on a set $M$, and let $S$ be any subset of $M$.

Let $M \times S$ be the class of all points of $M$ which are subsets of $S$. Then $M \times S$ satisfies Axioms I and II, and is thus a matroid on $S$. The flats of $\boldsymbol{M} \times S$ are those flats of $\boldsymbol{M}$ which are subsets of $S$. By the definition of dimension a flat $T$ of $\boldsymbol{M} \times S$ has the same dimension in $\boldsymbol{M} \times S$ as in $\boldsymbol{M}$. It is also clear that $T$ is connected in $\boldsymbol{M} \times S$ if and only if it is connected in $\boldsymbol{M}$.

Let $\boldsymbol{M}_{S}$ be the class of intersections with $S$ of members of $\boldsymbol{M}$, and let $\boldsymbol{M} \cdot \boldsymbol{S}$ be the class of all minimal non-null members of $\boldsymbol{M}_{S}$.

(3.1) If $X \in M$ and $a \in S \cap X$, then there exists $Z \in M \cdot S$ such that $a \in Z$ $\subseteq S \cap X$

Proof. We can find $Y \in M$ such that $a \in S \cap Y \subseteq S \cap X$ and $S \cap Y$ has the least number of elements consistent with this property. If $S \cap Y \in M \cdot S$ the theorem holds with $Z=S \cap Y$. In the remaining case there exists $Y^{\prime} \in M$ such that $\varnothing \subset S \cap Y^{\prime} \subset S \cap Y$ and, by the choice of $Y, a \notin Y^{\prime}$. Choose $c \in S \cap Y^{\prime}$. By Axiom II there exists $Y^{\prime \prime} \in M$ such that $a \in Y^{\prime \prime} \subseteq\left(Y \cup Y^{\prime}\right)-\{c\}$. But then $a \in S \cap Y^{\prime \prime} \subset S \cap Y \subseteq S \cap X$, contrary to the definition of $Y$. The theorem follows.

(3.2) $M \cdot S$ is a matroid on $S$.

Proof. By its definition $\boldsymbol{M} \cdot S$ satisfies Axiom I. Suppose $(X, Y) \in \boldsymbol{M} \cdot S$, $a \in X \cap Y$ and $b \in X-(X \cap Y)$. There exist elements $X^{\prime}$ and $Y^{\prime}$ of $\boldsymbol{M}$ such that $X=S \cap X^{\prime}$ and $Y=S \cap Y^{\prime}$. Since $M$ satisfies Axiom II there exists $U \in M$ such that $b \in U \subseteq\left(X^{\prime} \cup Y^{\prime}\right)-\{a\}$. By (3.1) there exists $Z \in M \cdot S$ such that $b \in Z \subseteq S \cap U \subseteq(X \cup Y)-\{a\}$. Thus $M \cdot S$ satisfies Axiom II.

(3.3) If $T$ is a flat of $\boldsymbol{M}$ then $S \cap T$ is a flat of $\boldsymbol{M} \cdot S$.

This follows from (3.1).

We define a carrier of $\boldsymbol{M} \cdot S$ in $\boldsymbol{M}$ as a subset $Z$ of $M$ such that $(\boldsymbol{M} \times Z) \cdot S$ $=M \cdot S$, and such that $d\langle Z\rangle$ has the least value consistent with this property. Such a carrier exists, since $(\boldsymbol{M} \times M) \cdot S=\boldsymbol{M} \cdot S$. We must have $S \subseteq Z$.

(3.4) If $Z$ is a carrier of $\boldsymbol{M} \cdot S$ in $\boldsymbol{M}$, then $d\langle Z-S\rangle=-1$ in $\boldsymbol{M}$.

Proof. Assume the theorem false. Then there exists $X \in M$ such that $X \subseteq Z-S$. Choose $a \in X$ and write $\boldsymbol{M}^{\prime}=(\boldsymbol{M} \times(Z-\{a\})) \cdot S$.

If $Y \in M^{\prime}$ there exists $U \in M \times Z$ such that $Y=S \cap U$. Hence there exists $Y^{\prime} \in(\boldsymbol{M} \times Z) \cdot S=\boldsymbol{M} \cdot S$ such that $Y^{\prime} \subseteq Y$. Conversely if $Y^{\prime} \in \boldsymbol{M} \cdot S=(\boldsymbol{M} \times Z) \cdot S$ there exists $V \in M \times Z$ such that $Y^{\prime}=S \cap V$. If $a \in V$ then by Axiom II there exists $U \in M \times Z$ such that $\varnothing \subset S \cap U \subseteq Y^{\prime}$ and $a \in U$. If $a \in V$ we write $U=V$. In each case $U \in M \times(Z-\{a\})$ and $S \cap U$ is non-null. Hence there exists $Y \in \boldsymbol{M}^{\prime}$ such that $Y \subseteq S \cap U \subseteq Y^{\prime}$. 
Applying Axiom $I$ to these results we find that $\boldsymbol{M}^{\prime}$ and $\boldsymbol{M} \cdot S$ are identical. But $d\langle Z-\{a\}\rangle=d\langle\langle Z\rangle-\{a\}\rangle\langle d\langle Z\rangle$, by I(2.3). Thus the definition of $Z$ as a carrier of $\boldsymbol{M} \cdot S$ is contradicted.

(3.5) Let $Z$ be a carrier of $M \cdot S$ in $M$. Then if $X \in M \cdot S$ there is just one $Y \in M \times Z$ such that $S \cap Y=X$.

Proof. Such a point $Y$ exists, since $\boldsymbol{M} \cdot S=(\boldsymbol{M} \times Z) \cdot S$. Suppose $Y^{\prime}$ is another such point of $M \times Z$. Choose $a \in S \cap Y=S \cap Y^{\prime}$. By Axiom I we can find $b \in Y^{\prime}-\left(Y^{\prime} \cap Y\right)$. By Axiom II there exists $U \in M \times Z$ such that $b \in U$ $\subseteq\left(Y \cup Y^{\prime}\right)-\{a\}$. Now $S \cap U \subset S \cap Y=X$. Hence $S \cap U$ is null, since $\boldsymbol{M} \cdot S$ satisfies Axiom I. Hence $U \subseteq Z-S$. This is impossible, by (3.4).

Given a carrier $Z$ of $\boldsymbol{M} \cdot S$ in $\boldsymbol{M}$ we define a mapping $\theta$ of the flats of $\boldsymbol{M} \cdot S$ into flats of $M \times Z$ in the following way:

(i) If $T$ is the null flat of $\boldsymbol{M} \cdot S$ then $\theta T$ is the null flat of $M \times Z$,

(ii) If $T$ is a point of $M \cdot S$ then $\theta T$ is the unique point $Y$ of $M \times Z$ such that $S \cap Y=T$,

(iii) If $T$ is a $d$-flat of $M \cdot S$, where $d>1$, then $\theta T$ is the union of all points $\theta X$ of $\boldsymbol{M} \times Z$ such that $X \subseteq T$. We refer to $\theta$ as the $(S, Z)$-mapping of $M$. We note that if $T$ is any flat of $M \cdot S$, then $T=\theta(T) \cap S$.

(3.6) If $T$ and $U$ are flats of $\boldsymbol{M} \cdot S$ then $\theta T \subseteq \theta U$ if and only if $T \subseteq U$.

This follows at once from the definition of $\theta$. It implies that $\theta T$ and $\theta U$ are distinct if $T$ and $U$ are distinct, and that $\theta T \subset \theta U$ if and only if $T \subset U$.

(3.7) If $V$ is a flat of $M \times Z$ then $V \subseteq \theta(S \cap V)$.

Proof. By (3.3) and the definition of $\theta$ we have $S \cap V \subseteq \theta(S \cap V)$.

If $b$ is any given cell of $V-(S \cap V)$ we can find $X_{b} \in M \times Z$ such that $b \in X_{b}$ and $S \cap X_{b} \subseteq S \cap V$. Choose such an $X_{b}$ so that $S \cap X_{b}$ has the least possible number of elements. Then $S \cap X_{b}$ is non-null, by (3.4).

Assume $S \cap X_{b} \in \boldsymbol{M} \cdot S$. There exists $Y \in M \cdot S$ such that $Y \subset S \cap X_{b}$. Choose $a \in Y$. We have $b \in \theta Y$, by the choice of $X_{b}$. By Axiom II we can find $Y^{\prime} \in M \times Z$ such that $b \in Y^{\prime} \subseteq\left(X_{b} \cup \theta Y\right)-\{a\}$. But then $S \cap Y^{\prime} \subset S \cap X_{b}$ and the definition of $X_{b}$ is contradicted.

We deduce that $S \cap X_{b} \in \boldsymbol{M} \cdot S$. Hence $b \in \theta\left(S \cap X_{b}\right) \subseteq \theta(S \cap V)$. Since this is true for all $b \in V-(S \cap V)$ we have $V-(S \cap V) \subseteq \theta(S \cap V)$. But $S \cap V$ $\subseteq \theta(S \cap V)$. The theorem follows.

(3.8) If $T$ is a flat of $\boldsymbol{M} \cdot S$ then $d T=d \theta T$.

Proof. If possible choose $T$ so that the theorem fails and $d T$ has the least value consistent with this. Clearly $T$ is non-null. Choose $a \in T$. Let $U$ be the union of all points of $M \cdot S$ which are subsets of $T-\{a\}$.

Now $\theta U$ is a subset of the flat $\langle\theta T-\{a\}\rangle$ of $M \times Z$, and $S \cap\langle\theta T-\{a\}\rangle \subseteq U$ by (3.3). Hence $\theta U \subseteq\langle\theta T-\{a\}\rangle \subseteq \theta(S \cap\langle\theta T-\{a\}\rangle) \subseteq \theta U$, by (3.6) and (3.7), and therefore $\theta U=\langle\theta T-\{a\}\rangle$. Now $d U=d T-1$ in $M \cdot S$ and $d\langle\theta T-\{a\}\rangle$ $=d \theta T-1$ in $M \times Z$, by I (2.3). But $d U=d \theta U=d\langle\theta T-\{a\}\rangle$, by the choice of $T$. Hence $d T=d \theta T$, contrary to the choice of $T$.

4. Binary and regular matroids. Let $N$ be a chain-group on a set $M$ over the ring $R$. If $f \in N$ we define the restriction $f \cdot S$ of $f$ to a subset $S$ of $M$ as 
that chain on $S$ in which each $a \in S$ has the same coefficient as in $f$.

We write $N \cdot S$ for the class of restrictions to $S$ of the chains of $N$, and $N \times S$ for the class of restrictions to $S$ of those chains $f$ of $N$ for which $|f| \subseteq S$. It is clear that $N \cdot S$ and $N \times S$ are chain-groups on $M$ over $R$ which satisfy the two following identities.

$$
\begin{aligned}
& \boldsymbol{M}(N \times S)=\boldsymbol{M}(N) \times S, \\
& \boldsymbol{M}(N \cdot S)=\boldsymbol{M}(N) \cdot S .
\end{aligned}
$$

It is shown in $[2,(3.5)]$, that $N \cdot S$ and $N \times S$ are regular if $N$ is regular. Hence (4.1) and (4.2) imply

(4.3) Let $M$ be a binary (regular) matroid on a set $M$, and suppose $S \subseteq M$. Then $\boldsymbol{M} \times S$ and $\boldsymbol{M} \cdot S$ are binary (regular) matroids.

(4.4) Every regular matroid is binary.

Proof. Let $\boldsymbol{M}$ be a regular matroid on a set $M$. There is a regular chaingroup $N$ on $M$ such that $\boldsymbol{M}=\boldsymbol{M}(N)$. If $f \in N$ let $\phi f$ be the chain on $M$ obtained by replacing each coefficient in $f$ by its residue mod 2. The chains $\phi f$ such that $f \in N$ constitute a binary chain-group $N^{\prime}$ on $M$.

Suppose $X \in M$. There is a primitive chain $g$ of $N$ such that $|g|=X$. Then $|\phi g|=X$. Hence there exists $X^{\prime} \in M\left(N^{\prime}\right)$ such that $X^{\prime} \subseteq X$. Conversely suppose $X^{\prime} \in \boldsymbol{M}\left(N^{\prime}\right)$. There is a chain $f \in N$ such that $|\phi f|=X^{\prime}$. There is a chain $h \in N$ which is a 2-representative of $f$. (See $[2, \S 6]$.) Then $|h|=|\phi f|=X^{\prime}$. Hence there exists $X \in M$ such that $X \subseteq X^{\prime}$. Using Axiom I we deduce from these results that $\boldsymbol{M}=\boldsymbol{M}\left(N^{\prime}\right)$. Hence $\boldsymbol{M}$ is binary.

By a figure of Type BI in a binary matroid $M$ we mean an assemblage of flats of $M$ consisting of a plane $P$, seven distinct lines on $P$, and the points of intersection of these lines. If $X$ is any point on $P$ there is a line $L$ on $P$ which is not on $X$, by $\mathrm{I}(2.4)$. There are at most three points on $L$, by (2.7). Hence at most three lines on $P$ are on $X$. It follows that each line of the figure is on three distinct points, each of which is on two other lines of the figure, and there can be no eighth line on $P$. By I(3.2) the seven lines are connected and therefore $P$ is connected. The seven lines intersect in threes at seven points and, by $\mathrm{I}(3.4)$, there can be no eighth point on $P$.

We enumerate the seven lines as $L_{1}, \cdots, L_{7}$, and denote the point of intersection, if any, of three lines $L_{i}, L_{j}$ and $L_{k}$ by $X_{i j k}$. We may assume a point $X_{135}$ on $L_{1}, L_{3}$ and $L_{5}$. The line $L_{7}$, which is not on $X_{135}$, meets $L_{1}, L_{3}$ and $L_{5}$ in points $X_{147}, X_{367}$ and $X_{257}$. The lines $L_{2}$ and $L_{4}$ each meet $L_{3}$ in its point other than $X_{135}$ and $X_{367}$, which is therefore $X_{234}$. Similarly the remainings two points of the figure are $X_{456}$ and $X_{126}$.

We can construct a matroid $M$ having a figure of Type BI on any set $S=\left\{a_{1}, \cdots, a_{7}\right\}$ of seven elements. The rule is that $X_{i j k}$ is the set of all elements of $S$ other than $a_{i}, a_{j}$ and $a_{k}$, and the $X_{i j k}$ are the only points of $\boldsymbol{M}$. Axioms I and II are easily verified. We can verify also that the chains mod 2 on $S$ whose domains are the $X_{i j k}$ generate a binary chain-group on $S$ whose 


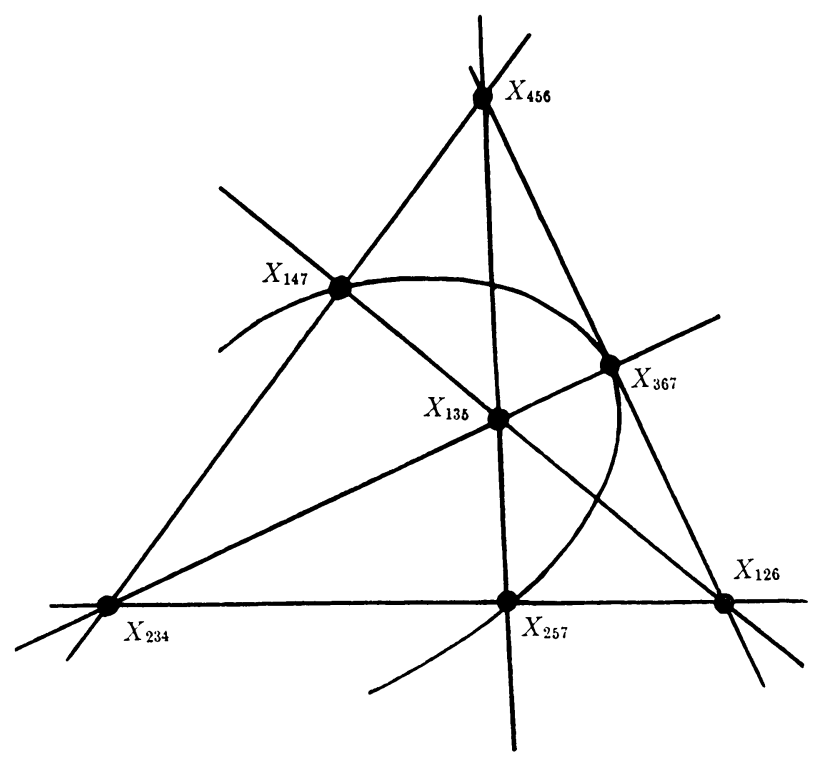

FIG. I

matroid is $M$. The lines of $M$ are the sets $L_{i}=S-\left\{a_{i}\right\}$ and $S$ is the only plane of $M$.

By a figure of Type BIl in a binary matroid $M$ we mean an assemblage of flats of $\boldsymbol{M}$ consisting of a 3 -flat $E$, seven distinct planes of $\boldsymbol{M}$ on $E$ such that no three are on a common line, the 21 lines of intersection of these planes, and those points of $E$ which are common to three or more of the seven planes.

We enumerate the seven planes as $P_{1}, \cdots, P_{7}$ and write $L_{i j}$ for the line of intersection of $P_{i}$ and $P_{j}(0 \leqq i<j \leqq 7)$.

By the argument already used for Type BI a point on the line $L_{i j}$ can be on at most three lines on the plane $P_{i}$. It follows that each of the lines $L_{i j}$ is on just three points of the figure, of which one is on just three of the seven planes and each of the others on just four of them. Hence each of the lines $L_{i j}$ is connected. It follows that the planes $P_{i}$ and the flat $E$ are all connected.

We write $X_{i j k}$ for a point which is on just three planes $P_{i}, P_{j}$ and $P_{k}$ of the figure, and $X_{i j k l}$ for a point on just four such planes $P_{i}, P_{j}, P_{k}$ and $P_{l}$. Each point of the first kind will be on just three lines of the figure, and each point of the second kind on just six. It follows that the figure has just seven points of each kind.

We obtain a detailed notation as follows. The line $L_{17}$ may be supposed on $X_{147}, X_{1237}$ and $X_{1567}$. The line $L_{47}$ is on two points $Y$ and $Z$ distinct from $X_{147}$. On $P_{7}$ each of these is on three lines of the figure meeting $L_{17}$ in three 
distinct points. Hence we may suppose $Y$ to be on $L_{27}$ and $L_{67}$ while $Z$ is on $L_{37}$ and $L_{57}$. Then $Y$ is $X_{2467}$ and $Z$ is $X_{3457}$. The remaining points of the figure on $P_{7}$ are $X_{367}=\left\langle L_{37} \cap L_{67}\right\rangle$ and $X_{257}=\left\langle L_{27} \cap L_{57}\right\rangle$. The lines $L_{13}$ and $L_{14}$ on $P_{1}$ meet in a point $X_{134 x}$, since $L_{14}$ is on $X_{147}$. Since no three planes of the figure are on a common line this point cannot have just three suffices in common with any of the points of the figure on $P_{7}$. It is therefore $X_{1346}$. But $X_{1346}$ is on the lines $L_{13}, L_{14}, L_{16}, L_{34}, L_{36}$ and $L_{46}$. These meet $P_{7}$ in $X_{1237}, X_{147}, X_{1567}$, $X_{3457}, X_{367}$ and $X_{2467}$ respectively. The third points on these lines are therefore $X_{135}, X_{1245}, X_{126}, X_{234}, X_{2356}$ and $X_{456}$. We have now accounted for all 14 points of the figure.

We may illustrate this structure in a modification of Figure I of Paper I. The points represented by four-pointed stars are considered to lie on $P_{7}$ and are accordingly given the extra suffix $7 . Z_{1}, Z_{2}$ and $Z_{3}$ are $X_{2356}, X_{1346}$ and $X_{1245}$ respectively. The disconnected lines $Z_{1} \cup Z_{2}, Z_{2} \cup Z_{3}$ and $Z_{3} \cup Z_{1}$ become the connected lines $L_{36}, L_{14}$ and $L_{25}$ respectively, meeting $P_{7}$ in new points $X_{367}, X_{147}$ and $X_{257}$.

We can construct a matroid $\boldsymbol{M}^{\prime}$ having a figure of Type BII on the set $S=\left\{a_{1}, \cdots, a_{7}\right\}$. The rule is that a point $Z$ of the figure is represented by the set of all elements of $S$ with suffices other than those of $Z$. It may be verified that the resulting 14 sets constitute a binary matroid $\boldsymbol{M}^{\prime}$ of the required kind on $S$. Moreover each of the two binary chain-groups on $S$ corresponding to $\boldsymbol{M}$ and $\boldsymbol{M}^{\prime}$ is the dual of the other.

(4.5) A matroid is regular if and only if it is binary and includes no figure of Type BI or BII.

Proof. Let $M$ be a regular matroid on a set $M$. There is a regular chaingroup $N$ on $M$ such that $\boldsymbol{M}=\boldsymbol{M}(N)$. $\boldsymbol{M}$ is binary, by (4.4).

Suppose $M$ includes a figure of Type BI. For each line $L_{i}$ of the figure we choose $a_{i} \in P-L_{i}$. Then $L_{i}=\left\langle P-\left\{a_{i}\right\}\right\rangle$ for each $L_{i}$, and the seven cells $a_{i}$ are distinct. There are primitive chains $g_{1}, g_{2}$ and $g_{3}$ of $N$ such that $\left|g_{1}\right|$ $=X_{234},\left|g_{2}\right|=X_{135}$ and $\left|g_{3}\right|=X_{126}$. Since each of these chains may be replaced by its negative we may suppose $g_{1}\left(a_{1}\right)=g_{2}\left(a_{2}\right)=g_{3}\left(a_{3}\right)=1$.

We note that $g_{i}\left(a_{j}\right)=0$ if $1 \leqq(i, j) \leqq 3$ and $i \neq j$. By (2.4) the set $\left\{a_{1}, a_{2}, a_{3}\right\}$ $=D$ say, meets the domain of every nonzero chain of $N \times P$. Clearly no proper subset of $D$ has this property.

In the terminology of $[2, \S 4], D$ is a dendroid of $N \times P$. This chain-group is regular, by $[2,(3.5)]$. The representative matrix of $N \times P$ associated with $D$ has the representative vectors of the chains $g_{i} \cdot P$ as its rows. If its first seven columns are taken to correspond to the seven cells $a_{i}$, in the order of the suffices, we may write them as

$$
\left\{\begin{array}{lllllll}
1 & 0 & 0 & 0 & e_{15} & e_{16} & e_{17} \\
0 & 1 & 0 & e_{24} & 0 & e_{26} & e_{27} \\
0 & 0 & 1 & e_{34} & e_{35} & 0 & e_{37}
\end{array}\right\}
$$


where each of the numbers $c_{i j}$ is 1 or -1 . By [2, (4.6)] we have

$$
\begin{gathered}
e_{16} e_{27}=e_{17} e_{26} \\
e_{24} e_{37}=e_{27} e_{34} \\
e_{35} e_{17}=e_{37} e_{15} \\
\left|\begin{array}{lll}
0 & e_{15} & e_{16} \\
e_{24} & 0 & e_{26} \\
e_{34} & e_{35} & 0
\end{array}\right|=e_{16} e_{24} e_{35}+e_{15} e_{26} e_{34}=0 .
\end{gathered}
$$

But these equations are contradictory, for they imply $-1=\left(e_{16} e_{24} e_{35}\right)$ $\cdot\left(e_{15} e_{26} e_{34}\right) e_{17}^{2} e_{27}^{2} e_{37}^{2}=\left(e_{16} e_{27} e_{17} e_{26}\right)\left(e_{24} e_{37} e_{27} e_{34}\right)\left(e_{35} e_{17} e_{37} e_{15}\right)=1$.

Suppose next that $\boldsymbol{M}$ includes a figure of Type BII. We proceed as before, choosing $a_{i} \in E-P_{i}$ and taking four primitive chains $g_{i}$ of $N$ satisfying $\left|g_{1}\right|$ $=X_{1567},\left|g_{2}\right|=X_{2467},\left|g_{3}\right|=X_{3457}$ and $\left|g_{4}\right|=X_{456}$. As the first seven columns of a representative matrix of the regular chain-group $N \times E$ associated with the dendroid $\left\{a_{4}, a_{5}, a_{6}, a_{7}\right\}$ we then obtain

$$
\left\{\begin{array}{lllllll}
0 & e_{24} & e_{34} & 1 & 0 & 0 & 0 \\
e_{15} & 0 & e_{35} & 0 & 1 & 0 & 0 \\
e_{16} & e_{26} & 0 & 0 & 0 & 1 & 0 \\
e_{17} & e_{27} & e_{37} & 0 & 0 & 0 & 1
\end{array}\right\},
$$

where each of the numbers $e_{i j}$ is 1 or -1 . Applying [2, (4.6)] we obtain the same contradictory equations as before.

We have now proved that if a matroid is regular then it is binary and includes no figure of Type BI or BII.

Assume the theorem false. Then there exists a matroid $M$ on a set $M$ which is binary and which includes no figure of Type BI or BII, but which is not regular. Choose such a matroid so that the number $\alpha(\boldsymbol{M})$ of its cells is as small as possible. Clearly $\alpha(\boldsymbol{M}) \geqq 1$. Choose $a \in M$ and write $\boldsymbol{M}^{\prime}=\boldsymbol{M}$ . $(M-\{a\})$. Then $\boldsymbol{M}^{\prime}$ has a carrier $Z$ in $\boldsymbol{M}$, and $Z$ is either $M-\{a\}$ or $M$. Let $\theta$ be the $(M-\{a\}, Z)$-mapping of $\boldsymbol{M}$.

$M^{\prime}$ is binary, by (4.3). If it includes a figure of Type BI or Type BII then the mapping $\theta$ induces another such figure in $\boldsymbol{M}$, which is impossible by the choice of $\boldsymbol{M}$. Since $\alpha\left(\boldsymbol{M}^{\prime}\right)<\alpha(\boldsymbol{M})$ we deduce that $\boldsymbol{M}^{\prime}$ is regular. Hence there is a regular chain-group $N^{\prime}$ on $M-\{a\}$ such that $\boldsymbol{M}^{\prime}=\boldsymbol{M}\left(N^{\prime}\right)$. For each $X \in M^{\prime}$ we select a primitive chain $g(X)$ of $N^{\prime}$ such that $|g(X)|=X$. For each $b \in M-\{a\}$ we denote the coefficient of $b$ in $g(X)$ by $s(X, b)$. Then $s(X, b)$ is 1 or -1 if $b \in X$ and zero otherwise.

Let $C$ denote the class of all points $T$ of $\boldsymbol{M}^{\prime}$ such that $a \notin \theta T$. Suppose $X, Y$ and $Z$ are distinct points of $\boldsymbol{M}^{\prime}$ on a line $L, X$ and $Y$ being members of C. Then $\theta X, \theta Y$ and $\theta Z$ are distinct points of $M$ on $\theta L$, and $\theta L$ is a connected 
line of $\boldsymbol{M}$, by (3.8) and I(3.2). Hence $a \notin \theta X \cup \theta Y=\theta L$, by I(3.1). Hence $a \notin \theta Z$, that is $Z \in C$. It follows that $C$ is a convex subclass of $\boldsymbol{M}^{\prime}$.

Let $X$ and $Y$ be distinct points of $\boldsymbol{M}^{\prime}-C$ on the same connected line $L$. By I(3.1) there exists $d \in X \cap Y$. We write

$$
t(X, Y)=s(X, d) s(Y, d) .
$$

The number $t(X, Y)$ thus defined is unique. For suppose $e$ is a second member of $X \cap Y$. By Axiom I we can find $b \in X-(X \cap Y)$ and $c \in Y-(X \cap Y)$. Using (2.4) we find that the representative vectors of $g(X)$ and $g(Y)$ are the rows of a representative matrix $K$ of $N^{\prime} \times L$ associated with the dendroid $\{b, c\}$. If the first four columns of $K$ are taken to correspond, in order, to $b, c, d$ and $e$ we may write them as

$$
\left\{\begin{array}{llll}
1 & 0 & s(X, d) & s(X, e) \\
0 & 1 & s(Y, d) & s(Y, e)
\end{array}\right\}
$$

Now $N^{\prime} \times L$ is regular, by $[2,(3.5)]$. Hence, by $[2,(4.6)]$ we have $s(X, d) s(Y, e)$ $-s(X, e) s(Y, d)=0$. Since each of the numbers concerned is 1 or -1 we may rewrite this as $s(X, d) s(Y, d)=s(X, e) s(Y, e)$.

If $R=\left(X_{1}, \cdots, X_{k}\right)$ is any nondegenerate path off $\boldsymbol{C}$ in $\boldsymbol{M}^{\prime}$ we write

$$
u(R)=\prod_{i=1}^{k-1} t\left(X_{i}, X_{i+1}\right)
$$

We proceed to show that $u(R)=1$ whenever $R$ is re-entrant.

We first note that for any re-entrant path $R$ off $\boldsymbol{C}$ in $\boldsymbol{M}^{\prime}$ there is a finite integer $m(R)$ which is the least number of elementary deformations with respect to $C$ by which $R$ can be converted into a degenerate path. This follows from the homotopy theorem of Paper I.

If possible choose a nondegenerate re-entrant path $R$ off $\boldsymbol{C}$ in $\boldsymbol{M}^{\prime}$ such that $u(R) \neq 1$ and $m(R)$ has the least value consistent with this. If $m(R)>1$ we can write either $R=R_{1} Q R_{2}$ and $R^{\prime}=R_{1} R_{2}$ or $R^{\prime}=R_{1} Q R_{2}$ and $R=R_{1} R_{2}$, where $O$ is an elementary re-entrant path with respect to $C$ and $m\left(R^{\prime}\right)=m(R)-1$. In each case $u(R)=u(Q) u\left(R^{\prime}\right)$. This is impossible since the choice of $R$ implies $u(Q) u\left(R^{\prime}\right)=1$. We deduce that $m(R)=1$, that is $R$ is an elementary re-entrant path with respect to $C$. $=1$.

If $R$ is of the first kind we may write $R=(X, Y, X)$. Then $u(R)=(t(X, Y))^{2}$

If $R$ is of the second kind we may write $R=(X, Y, Z, X)$, where $X, Y$ and $Z$ are distinct points on the same line or plane.

If $X, Y$ and $Z$ are on the same line $L$ then $\theta X, \theta Y$ and $\theta Z$ are distinct points of $\boldsymbol{M}$ on the same line $\theta L$ of $M$. But then $a \in \theta X \cap \theta Y \cap \theta Z$ and therefore $\theta L$ has a fourth point $\langle\theta L-\{a\}\rangle$. This is impossible, by (2.7).

We may now suppose that $X, Y$ and $Z$ are on a common plane $P$ of $\boldsymbol{M}^{\prime}$ but not on a common line. Hence there exist cells $b, c$ and $d$ of $P$, each be- 
longing to just one of the points $X, Y$ and $Z$, such that $b \in X, c \in Y$ and $d \in Z$.

If there is a cell $h$ of $P$ such that $h \in X \cap Y \cap Z$ we have $u(R)$ $=(s(X, h) s(Y, h))(s(Y, h) s(Z, h))(s(Z, h) s(X, h))=1$, contrary to the choice of $R$. Hence there are cells $e, f$ and $g$ of $P$, each belonging to just two of the points $X, Y$ and $Z$, such that $e \notin X, f \notin Y$ and $g \notin Z$.

If $x$ is any one of $b, c, d, e, f$ and $g$ we write $L_{x}$ for the line $\langle P-\{x\}\rangle$. Clearly the six lines $L_{x}$ of $\boldsymbol{M}^{\prime}$ on $P$ are all distinct. Hence the six lines $\theta L_{x}$ on the plane $\theta P$ of $M$ are all distinct. But each is on one of the points $\theta X$, $\theta Y$ and $\theta Z$, and therefore includes the cell $a$. Hence there is a seventh distinct line $\langle\theta P-\{a\}\rangle$ on $\theta P$. Thus $M$ includes a figure of Type BI, contrary to its definition.

If $R$ is of the third kind we have $R=(X, Y, Z, T, X)$, where $X, Y, Z$ and $T$ are distinct points on the same plane $P$, of which no three are on a common line. We can find cells $b, c, d$ and $e$ of $P$ such that $b \in P-(X \cup Y)$, $c \in P-(Y \cup Z), d \in P-(Z \cup T)$ and $e \in P-(T \cup X)$. Then $b$ belongs to $Z$ and $T$ but not to $X$ or $Y, c$ belongs to $T$ and $X$ but not to $Y$ or $Z$, and so on. The four chains $g(X), g(Y), g(Z)$ and $g(T)$ are linearly dependent, by (2.4). Hence

$$
\left|\begin{array}{cccc}
0 & 0 & s(Z, b) & s(T, b) \\
s(X, c) & 0 & 0 & s(T, c) \\
s(X, d) & s(Y, d) & 0 & 0 \\
0 & s(Y, e) & s(Z, e) & 0
\end{array}\right|=0
$$

that is $s(Z, b) s(T, c) s(X, d) s(Y, e)-s(T, b) s(X, c) s(Y, d) s(Z, e)=0$. It follows that $u(R)=(s(X, d) s(Y, d))(s(Y, e) s(Z, e))(s(Z, b) s(T, b))(s(T, c) s(X, c))=1$.

If $R$ is of the fourth kind we write $R=(A, X, B, Y, A)$ and then use the notation set out in $\mathrm{I}, \S 5$ for this case. We observe that no three of the planes $P_{i}$ on $E$ have a common line, that each is on just two points of $\boldsymbol{C}$, and that no two of the planes $P_{i}$ have more than one point of $C$ in common. It follows that on the 3-flat $\theta E$ of $M$ the seven planes $\theta P_{1}, \cdots, \theta P_{6},\langle\theta E-\{a\}\rangle$ are all distinct and that no three of them are on a common line. But then $M$ includes a figure of Type BII, contrary to its definition.

For each kind of elementary re-entrant path we have obtained a contradiction. We deduce that $u(R)=1$ whenever $R$ is a nondegenerate re-entrant path off $\boldsymbol{C}$ in $\boldsymbol{M}^{\prime}$.

We can partition the points of $\boldsymbol{M}^{\prime}-\boldsymbol{C}$ into disjoint non-null classes $K_{1}, \cdots, K_{q}$ such that for any two points $X$ and $Y$ of $\boldsymbol{M}^{\prime}-C$ there is a path from $X$ to $Y$ off $C$ in $\boldsymbol{M}^{\prime}$ if and only if $X$ and $Y$ belong to the same class $K_{i}$. For each class $K_{i}$ we select an arbitrary representative $T_{i}$ and write $v\left(T_{i}\right)=1$. For any other point $X$ in $K_{i}$ we write

$$
v(X)=u(Q) v\left(T_{i}\right)
$$


where $Q$ is any path from $T_{i}$ to $X$ off $C$ in $M^{\prime}$. If $Q^{\prime}$ is any other such path we have $u(Q) u\left(Q^{\prime}\right)=u\left(Q^{-1}\right) u\left(Q^{\prime}\right)=u\left(Q^{-1} Q^{\prime}\right)=1$, since $Q^{-1} Q^{\prime}$ is re-entrant. Hence the number $v(X)$ is uniquely defined for each $X \in \boldsymbol{M}^{\prime}-\boldsymbol{C}$ when the representatives $T_{i}$ have been chosen.

For each $U \in M$ we define a chain $f_{U}$ on $\boldsymbol{M}$ over the ring of integers as follows.

Suppose $a \in U$. If $U=\{a\}$ we write $f_{U}(a)=1$ and $f_{U}(b)=0$ if $b \neq a$. If $U \not \neq\{a\}$ then $U-\{a\}$ is a non-null flat of $\boldsymbol{M}^{\prime}$, by (3.3). The flat $\theta(U-\{a\})$ of $M \times Z$ is $U-\{a\}$ or $U$, and the former alternative is ruled out by Axiom I. Hence $U-\{a\}$ is a point $X$ of $\boldsymbol{M}^{\prime}-\boldsymbol{C}$, by (3.8). We choose $f_{U}$ to agree with $g(X)$ in $M-\{a\}$ and to satisfy $f_{U}(a)=v(X)$.

Suppose $a \notin U$. Then $U$ is a flat of $\boldsymbol{M}^{\prime}$, by (3.3), and $\theta U=U$ or $U \cup\{a\}$. In the former case $U$ is a point of $\boldsymbol{M}^{\prime}$, by (3.8). We then choose $f_{U}$ to agree with $g(U)$ in $M-\{a\}$ and to satisfy $f_{U}(a)=0$. In the latter case $U \cup\{a\}$ is a line of $\boldsymbol{M}$, by $\mathrm{I}(2.3)$, and $U$ is a line of $\boldsymbol{M}^{\prime}$, by (3.8). Now each point of $\boldsymbol{M}^{\prime}$ on $U$ is mapped by $\theta$ onto a point of $\boldsymbol{M}$ on $U \cup\{a\}$. But there are at most three points of $M$ on $U \cup\{a\}$, by (2.7), and one of these is $U$. Hence $U$ is a disconnected line of $\boldsymbol{M}^{\prime}$, having only two points $X$ and $Y$. Write $V=\theta X$ and $W=\theta Y$. Then $V=X \cup\{a\}$ and $W=Y \cup\{a\}$, by Axiom I. Thus $f_{V}$ and $f_{W}$ are already defined. We take $f_{V}$ to be one of the differences $f_{V}(a) f_{W}-f_{W}(a) f_{V}$ and $f_{W}(a) f_{V}-f_{V}(a) f_{W}$.

We observe that for each $U \in M$ we have $\left|f_{U}\right|=U$. Moreover $f_{U} \cdot(M-\{a\})$ is a chain of $N^{\prime}$.

Suppose $U, V$ and $W$ are the three points on a connected line $L$ of $\boldsymbol{M}$. We proceed to prove that $f_{U}, f_{V}$ and $f_{W}$ are linearly dependent.

We discuss first the case $a \in L$. We may suppose $a \in V \cap W$ and $a \in U$, by (2.6). Then there are points $X=V-\{a\}$ and $Y=W-\{a\}$ of $\boldsymbol{M}^{\prime}$ such that $\theta X=V$ and $\theta Y=W$. Write $L^{\prime}=L \cap(M-\{a\})=L-\{a\}$. Then $L^{\prime}$ is a flat of $M^{\prime}$, by (3.3). It clearly satisfies $\theta L^{\prime}=L$, and is therefore a line of $M^{\prime}$. The set $U$ is also a flat of $M^{\prime}$, by (3.3). It must be either the line $L^{\prime}$ or a point on it.

If $U$ is a point of $M^{\prime}$ on $L^{\prime}$ then $\theta U=U$, and $U$ is distinct from $X$ and $Y$. The line $L^{\prime}$ is then connected, by $\mathrm{I}(3.2)$. We can define $v(X)$ by a path $Q$ off $C$ from a representative point $T_{i}$ to $X$, and then define $v(Y)$ by the path $Q(X, Y)$. We then have

$$
v(X) v(Y)=u(Q) v\left(T_{i}\right) \times u(Q) t(X, Y) v\left(T_{i}\right)=t(X, Y) .
$$

Hence for each $b \in X \cap Y$ we have $v(X) v(Y)=s(X, b) s(Y, b)$. By (2.6) this implies

$$
|v(X) g(Y)-v(Y) g(X)| \subseteq U
$$

Since $g(U)$ is an elementary chain of $N^{\prime}$ it follows that

$$
g(U)= \pm(v(X) g(Y)-v(Y) g(X)) .
$$

By the definitions of $f_{U}, f_{V}$ and $f_{W}$ we now have 


$$
f_{U}= \pm\left(f_{V}(a) f_{W}-f_{W}(a) f_{V}\right) .
$$

If $U$ is the line $L^{\prime}$ of $\boldsymbol{M}^{\prime}$ we have $\theta U=U \cup\{a\}$, by (3.8). Then (4.5a) follows at once from the definition of $f_{U}$. Thus (4.5a) holds whenever $a \in L$, and the chains $f_{U}, f_{V}$ and $f_{W}$ are linearly dependent in this case.

Now suppose $a \notin L$. If $U, V$ and $W$ are points of $\boldsymbol{M}^{\prime}$ and $L$ is a line of $\boldsymbol{M}^{\prime}$ there is a linear relation between $g(U), g(V)$ and $g(W)$, by (2.4). A corresponding relation must hold between $f_{U}, f_{V}$ and $f_{W}$. In the remaining case $\theta L=L \cup\{a\}$. Then $\theta L$ is a connected plane of $M$, by $\mathrm{I}(2.3)$ and the fact that some point on $\theta L$ must meet both $L$ and $\{a\}$. By I(3.4) there are connected lines $L_{U}, L_{V}$ and $L_{W}$ on $\theta L$, distinct from $L$, whch are on $U, V$ and $W$ respectively. We can choose these lines to have no common point. For suppose $T$ is such a common point. There is a point $T^{\prime}$, distinct from $T$ and $U$, on $L_{U}$. By I(3.4) there is a connected line $L^{\prime}$, distinct from $L_{U}$, on $L$ and $T^{\prime}$. Without loss of generality we may suppose $\left\langle L \cap L^{\prime}\right\rangle=V$. Replacing $L_{V}$ by $L^{\prime}$ we obtain three lines of the kind required. Write $U^{\prime}=\left\langle L_{V} \cap L_{W}\right\rangle, V^{\prime}=\left\langle L_{W} \cap L_{U}\right\rangle$ and $W^{\prime}=\left\langle L_{U} \cap L_{V}\right\rangle$. We note that $a \in U^{\prime} \cap V^{\prime} \cap W^{\prime}$. Hence we can apply (4.5a) to the lines $L_{U}, L_{V}$ and $L_{W}$ and obtain

$$
\begin{aligned}
f_{U} & = \pm\left(f_{V^{\prime}}(a) f_{W^{\prime}}-f_{W^{\prime}}(a) f_{V^{\prime}}\right) \\
f_{V} & = \pm\left(f_{W^{\prime}}(a) f_{U^{\prime}}-f_{U^{\prime}}(a) f_{W^{\prime}}\right), \\
f_{W} & = \pm\left(f_{U^{\prime}}(a) f_{V^{\prime}}-f_{V^{\prime}}(a) f_{U^{\prime}}\right) .
\end{aligned}
$$

Thus $f_{U}, f_{V}$ and $f_{W}$ are linearly dependent in this case also.

The preceding analysis shows that the chains $f_{U}, U \in M$ satisfy the conditions of (2.5). They therefore generate a chain-group $N$ on $M$ such that $\boldsymbol{M}=\boldsymbol{M}(N)$. This implies that the $f_{U}$ are primitive chains of $N$, and hence that $N$ is regular. But then $M$ is regular, contrary to its definition. This contradiction establishes the theorem.

\section{REFERENCES}

1. C. Kuratowski, Sur le probleme des courbes gauches en Topologie, Fund. Math. vol. 15 (1930) pp. 271-283.

2. W. T. Tutte, $A$ class of Abelian groups, Canadian J. Math. vol. 8 (1956) pp. 13-28.

3. —_, A homotopy theorem for matroids, I. Trans. Amer. Math. Soc. vol. 86 (1958) pp. 144-160.

University OF TORONTO,

TORONTO, ONT. 